\title{
Estudo de efetividade de esquemas alternativos para o tratamento da tuberculose multirresistente no Brasil ${ }^{*}$
}

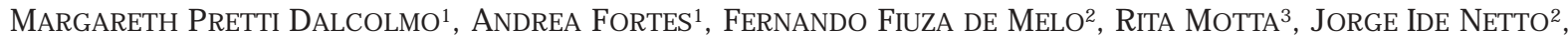 \\ Ninarosa Cardoso ${ }^{4}$, Monica Andrade 5 , Angela WerneCK Barreto ${ }^{1}$, Germano Gerhardi ${ }^{1}$
}

O bjetivo: Determinar a efetividade do tratamento com esquemas alternativos para casos confirmados de tuberculose multirresistente (TBMR) primária e adquirida, em pacientes ambulatoriais. Métodos: Casos de TBMR foram definidos como cultura e isolamento de $M$. tuberculosis e perfil de resistência in vitro a pelo menos à rifampicina, isoniazida e a uma terceira droga dos esquemas padronizados no Brasil, tanto pelo método convencional (LJ ) quanto pelo sistema radiométrico BACTEC. Desenho: Ensaio clínico, multicêntrico, não randomizado e controlado. Os pacientes foram arrolados entre abril de 1995 e dezembro de 1997, no total de 197. Por diversas razões 10 casos foram excluídos da análise. Em abril de 1998 permaneciam em tratamento 36 pacientes. Foram analisados 149 casos com duração média de tratamento de 14 meses sem interrupção.

Os regimes foram escolhidos conforme o perfil de sensibilidade: 1) estreptomicina, ofloxacina, terizidona, etambutol, clofazimina ou 2) amicacina, ofloxacina, terizidona, etambutol e clofazimina. Demografia: sexo: masculino - 68,4\%, feminino - 31,5\%; média de idade - 36,9 anos (14-71 anos); prevalência de HIV - 1,9\%; taxa de resistência primária - 8\%, taxa de resitência secundária - 92\%. Resultados parciais: 120 (79,5\%) pacientes negativaram a cultura no período de 3 meses; cura - 53\%, falência - 31\%, óbito - 6\%, abandono - 10\%. Definições: cura - tratado por 12 meses, com 6 meses de tratamento após 2 culturas consecutivas negativas; abandono - tratamento e consultas descontinuados; óbito - morte causada por TB após 2 meses de tratamento; falência - persistência de positividade na cultura em 12 meses seguidos. Conclusão: 0 maior preditor da multirresistência no estudo foi tratamento prévio irregular ou incompleto. Outros preditores $(p<0,05)$ foram: ser homem, ter lesão radiológica cavitária bilateral e ter mais de 2 anos de doença. A taxa de conversão bacteriológica em escarro e cultura foi alta em 6 meses de tratamento. (J Pneumol 1999;25(2):70-77)

\section{Outcomes of multidrug resistant tuberculosis (MDR TB) treatment in Brasil - Partial results - As of April, 1998}

Purpose: To determine the effectiveness of alternative regimens for treating confirmed MDR TB cases in outpatient units: Methods: MDR TB cases were defined as culture isolation of M. tuberculosis resistant in vitro to at least rifampin/ isoniazide and a third drug of standard regimens in Brazil, according to both the conventional method and the BACTEC system. Design: Multicentric non-randomized controlled clinical trial. From A pril 95 to December 97187 patients were enrolled. As of A pril 98, there were 36 patients under treatment. The authors analyzed 149 patients that had an average

14 months of treatment duration. The regimens used were chosen according to sensitivity tests: 1) streptomycin (S)/ ofloxacin (OFX)/terizidon (TZ)/ethambutol (E)/clofazimine (CZ) or capreomycin (CM)/OFX/TZ/CZ/E or amicacyn/OFX/TZ/E/CZ. Demography: Male, $68.4 \%$; female, $31.5 \%$; mean age-36.9 years; HIV prevalence 1.9\%; primary resistance rate - $8 \%$. Outcomes: cured-treated for 12 months with six months after two consecutive negative culture; abandoned-discontinued treatment and consultations; died - deaths due to TB after two months of treatment; failed - remained positive in culture throughout the 12 months. Results: 120 (79.5\%) patients converted to negative culture within 3 months. Cured: 53\%, failed 31\%, died 6\%, abandoned 10\%. Conclusions: The major predictor to MDR TB in Brazil is previous irregular and/or incomplete treatment. Sputum conversion rate was high and favorable overall response was $53 \%$. Clinical implications: Because TB is endemic in Brazil and the number of MDR cases due to acquired resistance is increasing, greater efforts are needed to implement supervised treatment in tuberculosis particularly for non compliant patients.

\footnotetext{
* Trabalho vencedor do II Prêmio Nacional de Pesquisa em Tuberculose, outorgado pela SBPT em 1998.

1. Médico do Centro de Referência Hélio Fraga, RJ .

2. Médico do Instituto Clemente Ferreira, SES, São Paulo.

3. Médica do Hospital Raphael de Paula Souza.

4. Médica do Hospital J oão de Barros Barreto, UFPA, Belém.

5. Médica do IDT - UFRJ .
}

Estudo financiado com grant do PNCT/MS e Centro de Referência Hélio Fraga - RJ.

Endereço para correspondência - Margareth Pretti Dalcolmo, Centro de Referência Hélio Fraga, Estr. de Curicica, 2.000, J acarepaguá - 22710-550 - Rio de J aneiro, RJ, e-mail: mdalcolmo@openlink.com. br

Recebido para publicação em 15/2/99. 
Descritores - Tuberculose, tratamento. Tuberculose multirresistente. Key words - Tuberculosis, treatment. Multidrug resistant tuberculosis.

\section{RATIONALE}

Em 1979 a Divisão Nacional de Pneumologia Sanitária/ Ministério da Saúde (DNPS/MS) normalizou para todo o país um Programa de Controle da Tuberculose (PCP) tendo como base fundamental o tratamento quimioterápico, com um esquema de primeira linha, o chamado Esquema 1 (E-1) - 2RHZI $4 \mathrm{RH}$, indicado para todas as formas de tuberculose sem uso prévio de medicação e, para o retratamento, nos casos de recidiva depois de curados ou que retornam positivos após abandonarem o tratamento inicial. Para os casos de falência a este regime, foi preconizado um esquema de reserva, o Esquema 3 (E-3) - 3SZME/ $9 \mathrm{ME}^{(1)}$.

Sendo a resistência primária às drogas em nosso país ainda considerada muito baixa ${ }^{(2,3)}, 0 \mathrm{E}-1$ deveria proporcionar uma eficácia aproximada de 98 a $99 \%$ e uma efetividade próxima dos $90 \%$. Porém, com a gradativa deterioração do Sistema de Saúde Pública no país, associada à piora das condições socioeconômicas da população, este tratamento vem apresentando resultados desfavoráveis, principalmente no que se refere às crescentes taxas de abandono observadas em centros urbanos de grande população e, secundariamente, pelo baixo potencial dos esquemas de retratamento.

Em 1992, uma avaliação do desempenho do PCP na década de 80 revelou os seguintes resultados do tratamento com o E-1 em condições de rotina: $79,8 \%$ de cura, com $12,9 \%$ de abandono, 3,3\% de óbito, 2,2\% de falência, $0,2 \%$ de mudança de drogas por efeitos colaterais e 1,5\% de transferência, de um total de 145.387 casos analisados. Dos que abandonam, cerca de $30 \%$ retornam positivos ao sistema para retratamento ${ }^{(4)}$.

O s resultados do retratamento com o E-1 quando comparado ao inicial são bem menores conforme um informe apresentado em 1992, demonstrando que a falência passa de $1,7 \%$ para $7,5 \%$, o que levou os autores, em reunião técnica subvencionada pelo Ministério da Saúde, em 1993, a sugerirem o acréscimo neste retratamento de uma quarta droga, o etambuto| $\left.\right|^{(5,6)}$.

São poucos os estudos de efetividade e rendimento do esquema de reserva, o E-3, no país. No Rio Grande do Sul, um estudo retrospectivo apresentado em 1990 mostrou $66,7 \%$ de cura, $17,9 \%$ de abandono, $8,2 \%$ de óbito e $7,2 \%$ de falência em 195 pacientes ${ }^{(7)}$. Recentemente, um comunicado pessoal de C.F.C. Rizzon no VIII Congresso Brasileiro de Infectologia, em setembro de 1994, estudando 108 pacientes em duas unidades da Grande Porto Alegre, encontrou $55,5 \%$ de cura, $17,6 \%$ de abandono, $25 \%$ de falência e $1,9 \%$ de óbito ${ }^{(8)}$, que foi semelhante a um estudo anterior de

\author{
Siglas e abreviaturas utilizadas neste trabalho \\ R - Rifampicina \\ $\mathrm{H}$ - Isoniazida \\ Z - Pirazinamida \\ S - Estreptomicina \\ M - Etambutol \\ E - Etionamida \\ TRZ - Terizidona \\ CFZ - Clofazimina \\ RFB - Rifabutina \\ OFX - Ofloxacina \\ CM - Capreomicina \\ TBMR - Tuberculose multirresistente
}

146 pacientes em uma unidade hospitalar entre, 83 e 87 , com respectivamente $61,6 \%, 19,2 \%, 10,3 \%$ e $8,9 \%{ }^{(9)}$. Estes resultados estão bem aquém dos encontrados no tratamento com o E-1, o que demonstra o baixo potencial do esquema de reserva ora em uso no país.

Este panorama, nos últimos anos, descortina um universo residual de pacientes que não obtêm cura após tratamento com OS E-1 e E-3 normalizados, em geral portadores de bacilos resistentes a mais de uma das drogas usuais no país e reconhecidos como portadores de tuberculose multirresistente (TBMR). Tomando por base as informações existentes sobre o rendimento do E-1, retratamento com E-1 e E-3, foi estabelecido um modelo para cálculo estimativo da incidência anual da TBMR: dos pacientes que iniciam o E-1(VT) 8,5\% irão ser retratados com este mesmo (RT), 4,05\% com o E-3(R) e 0,4\% necessitam de esquema alternativo $(M R)^{(10)}$.

Em relação ao total de casos notificados anualmente e pelo somatório acumulado dos que passam vários anos no sistema de saúde, o número de casos no país de pacientes com TBMR, conforme levantamento nacional realizado pelo Centro de Referência Prof. Hélio Fraga em final de 1995, revelou cerca de 1.500 casos, localizados, nos maiores números, no Rio de J aneiro e em São Paulo. Nestes dois Estados se concentram, respectivamente, 16.858 (18,5\%) e 18.266 (20\%) do total de casos no país (91.013 casos) e as taxas de abandono em suas capitais é de $30 \%$ e $20 \%$. Quanto aos pacientes soropositivos para HIV, estes são mais susceptíveis à infecção por cepas resistentes ${ }^{(11)}$. Este fato aumenta a preocupação com as situações dos Estados de Rio de J aneiro e São Paulo, porque, além de concentrarem mais da metade do número de pacientes portadores de TBMR, são também os de maior prevalência de casos de SIDA, favorecendo, assim, a ocorrência de eventuais epidemias de TBMR entre esses pacientes.

\section{INTRODUÇÃO}

O CDC (Centers for Disease Control), órgão de saúde pública norte-americano, em 1992, em publicação sobre a 
trajetória da tuberculose multirresistente no país, mostrou, de 1982-1986, 0,5\% de novos casos resistentes à INH e RMP. Em 1991 houve um aumento desta proporção para 3,1\%. Entre recidivas, $3 \%$ se mostraram resistentes a duas drogas durante 1982-1986 e, em 1991, esta proporção aumenta para 6,9\%. De 1990 até o início de 1992 o CDC, em colaboração com vários Estados e seus respectivos departamentos de saúde, investigaram vários casos de tuberculose multirresistente, notificados a partir de surtos institucionais (hospitais e prisões). Todos os casos eram resistentes à RMP e INH e alguns espécimes mostraram resistência a sete das drogas freqüentemente utilizadas. A maioria desses pacientes tinha co-infecção pelo HIV e a mortalidade foi muito elevada, 72 a $89 \%^{(12)}$.

O aumento do número de casos de TB nOS EUA com 28.000 casos de excesso registrados entre 1980 e 1992 suscitou o reconhecimento do problema como de saúde pública e o desenvolvimento, em dezembro de 1991, através da Federal Task Force, de um plano nacional de combate à tuberculose visando a erradicação da doença nas próximas décadas. Esse plano contemplou, de modo especial, a tuberculose resistente a múltiplas drogas, estabelecendo objetivos, cuidados e atividades a serem iniciados em 1992 e 1993, em cooperação dos órgãos de saúde com a sociedade civil. Independentemente de sabermos que erradicar não seja um conceito epidemiológico aplicável a uma doença de comportamento endêmico como a tuberculose, medidas como estas são positivas naquilo que favorecem o controle da doença ${ }^{(12)}$.

Mesmo sabendo de dados crescentes da incidência da tuberculose no nosso país e do acúmulo de crônicos (resistência in vitro aos esquemas preconizados e falência operacional ao tratamento), foram necessários a sinalização e o alarme feito pelos norte-americanos de uma suposta "nova" forma de apresentação da tuberculose (TBMR) para que o mundo e, em especial, os países europeus voltassem a se assustar e retomar ações de controle da doença.

Observe-se que a resistência bacteriana não se constitui em fenômeno novo ou uma nova forma da doença. Desde o aparecimento das primeiras drogas, como a estreptomicina, já se observava a presença de bacilos naturalmente resistentes. Igualmente, não se deve a novos mecanismos, mas a mutações genéticas já bem estabelecidas desde 0 início da década de $70^{(13-15)}$.

Com a deterioração dos programas de controle da tuberculose dos Estados e municípios, nas principais cidades de maior prevalência da doença, quer por distribuição irregular das drogas, quer por falta de atendimento adequado ao paciente com tuberculose, e ainda pelos poucos recursos laboratoriais, temos nos defrontado cada vez mais com pacientes mal assistidos e virtuais resistentes às drogas normalizadas.

A droga-resistência no Brasil está fundamentalmente associada a mau tratamento prévio. As formas de resistência às drogas antituberculosas são: primária - aquela observada em pacientes seguramente não tratados anteriormente, infectados por uma fonte doente com forma resistente; adquirida ou secundária - resultante de uso prévio de medicação de forma inadequada, seja por esquemas de baixa potência ou feitos por tempo insuficiente.

Realizado em nosso país recentemente, inquérito da resistência primária mostrou os seguintes resultados: a taxa de resistência primária nos pacientes VT (virgens de tratamento) foi de $9,2 \%$ a uma droga e de $-0,8 \%$ a duas drogas; nos pacientes RT (retratamento), foi de $21,8 \%$ a uma droga e de $5,7 \%$ a duas drogas. Ainda valores baixos, porém com aumento da resistência primária no retratamento(15-17).

Para estes pacientes não foram ainda consolidadas orientações normativas, embora já sejam reconhecidos como um problema e comecem a aparecer orientações genéricas sobre a questão por parte das instâncias ministeriais(4,18), especialmente após a emergência de surtos localizados de TBMR na América do Norte, que serviram para alertar e alardear para a gravidade do problema ${ }^{(12,19-22)}$.

A ssim, diferentemente do conceito internacional, em particular 0 aplicado na literatura norte-americana, que define a TBMR como uma doença com bacilos resistentes a $\mathrm{R}$ e $\mathrm{H}$ ou a mais de uma droga ${ }^{(12,23,24)}$, no Brasil, considerando os esquemas normalizados pela CNPS/MS, esta forma da tuberculose compreenderia os pacientes com doença ativa, portadores de bacilos resistentes a pelo menos 3 drogas: $\mathrm{R}, \mathrm{H}$ e mais $\mathrm{S}$ e/ ou $\mathrm{M}^{(6,18)}$. Diferem também dos "antigos crônicos" acumulados antes da introdução do PCP, em geral sensíveis ao E1 , por apresentarem bacilos resistentes aos mais poderosos medicamentos conhecidos até o momento e usuais no país, o que torna a sua abordagem terapêutica bastante complica$\mathrm{da}^{(6,25)}$. Devem ser agregados a este grupo, os pacientes, em geral contatos de portadores de TBMR, que apresentam resistência primária à $\mathrm{R}, \mathrm{H}$ e a outras droga usuais, geralmente a $\mathrm{S}$ e/ ou M.

Como perspectiva promissora para os portadores de TBMR, vêm surgindo novos fármacos e recuperados velhos tuberculostáticos, o que possibilita a formulação de esquemas terapêuticos alternativos. Entre os novos, destaque para os derivados quinolônicos e as novas ansamicinas; entre os recuperados, outros aminoglicosídeos e a ciclosserina e seus análo$\operatorname{gos}^{(26-28)}$.

Em 1993 foi realizado o 9o Fórum em Microbiologia sobre Emergência da Tuberculose Multidrogarresistente, estabelecidos e publicados no A nuário do Institut Pasteur.

A os pacientes com suspeita de TBMR, rever uso prévio de drogas e confirmação in vitro da sensibilidade. Baseado nisso, a proposta terapêutica deve se compor de, no mínimo, três medicamentos não utilizados anteriormente, com comprovada sensibilidade in vitro. Entre esses, prioritariamente, um agente oral do grupo das fluorquinolonas e um agente parenteral, aminoglicosídeo, nos primeiros meses do tratamento ${ }^{(15) .}$ 
Algumas experiências nacionais testando esquemas alternativos, porém com número limitado de pacientes ou com os resultados ainda não consolidados, apresentam respostas favoráveis que variam de 20 a $70 \%(6,29-31)$. A maioria destes trabalhos foi exclusivamente com medicamentos existentes no mercado nacional, e com pacientes quase que em sua maioria com resistência adquirida. Para analisá-los comparativamente há uma dificuldade adicional, qual seja, a metodologia não foi semelhante.

As experiências internacionais mostraram uma taxa de efetividade em torno de $54 \%{ }^{(32,33)}$, também com resistência adquirida.

Com a preocupação de estabelecer uma experiência brasileira para verificação de efetividade de tratamento alternativo para pacientes portadores de TBMR, é que os autores propuseram, para aprovação e financiamento, ao Ministério da Saúde, o presente estudo cooperativo, que conta com o concurso de algumas drogas importadas do mercado internacional e outras produzidas no país, sob a coordenação do Centro de Referência Prof. Hélio Fraga/MS - Rio de J aneiro e do Instituto Clemente Ferreira/ SES - São Paulo.

\section{OBJETIVOS DO ESTUdO}

\section{Geral:}

1) Determinar a efetividade de esquemas alternativos para pacientes portadores de TBMR.

\section{Específicos:}

1) Determinar a factibilidade de tratamento de portadores de TBMR nas condições de operação ora existentes no Brasil.

2) Testar intervenções, tais como: maior número de consultas e consultas de enfermagem, na adesão ao tratamento.

3) Avaliar os efeitos adversos e possíveis interações farmacológicas das drogas usadas.

4) Ampliar os conhecimentos sobre os pacientes portadores de TBMR e as características desta forma de apresentação da doença.

5) Contribuir, com base nos conhecimentos adquiridos, para o conceito e controle de TBMR no país.

6) Controlar o aparecimento de novos casos de TBMR através de controle dos contatos dos pacientes arrolados no estudo.

\section{MATERIAL E MÉTOdOS}

Desenho do estudo: Ensaio clínico prospectivo, multicêntrico, controlado e não randomizado. A randomização, neste caso, estaria fora de cogitação, dado que os casos arrolados serão incluídos nos esquemas de acordo com sua sensibilidade. 0 tratamento será quimioterápico, principalmente ambulatorial e auto-administrado, com consultas se- manais e quinzenais, recomendando-se a supervisão, direta ou indireta, em alguns casos, particularmente na fase inicial, e a internação para a supervisão na fase de ataque ou por razões clínicas - as mesmas recomendadas pelas normas do $\mathrm{PCT}$, por um prazo não superior a 30 dias.

Centros participantes: 0 estudo será desenvolvido nos centros a seguir designados, a partir de pacientes matriculados nos seus serviços ambulatoriais ou encaminhados pela rede pública, segundo os critérios de inclusão. a) Rio de J aneiro: centros número 1 e 2 (H élio Fraga/ HRPS), b) São Paulo: centro número 3 (ICF-SES), C) Belém: centro número 4 (Hosp. J BB-UFPA).

Tamanho da amostra: Serão arrolados, no estudo, 180 a 200 pacientes selecionados em dois grupos, de acordo com a sensibilidade às drogas observada nos testes de laboratório. A distribuição dos grupos por centro será desigual, estando relacionada à demanda de casos virtualmente candidatos.

Aprovação ética: 0 estudo foi submetido e aprovado pelos Comitês de Bioética do Conselho Nacional de Saúde e pelo CRM do Estado do Rio de J aneiro, com a chancela do CFM.

\section{Critérios de inclusão e exclusão de pacientes:}

Critérios de inclusão:

a) Pacientes que fizeram uso de E-1 e de E-2, com falência a ambos, apresentando resistência bacteriológica a pelo menos três drogas (3) dos esquemas padronizados, ou seja, portadores de tuberculose pulmonar ativa, comprovada por cultura e tipificação para o Mycobacterium tuberculosis no exame de escarro.

b) Contatos de pacientes MR que apresentam resistência micobacteriana com o mesmo perfil acima.

c) Pacientes com provas de função hepática e renal compatíveis com o uso das drogas, respectivamente: transaminases abaixo de três vezes os valores normais e creatinina abaixo de $2,0 \mathrm{mg} / \mathrm{dl}$.

d) Pacientes que concordem em participar do estudo, assinando termo de consentimento respectivo.

Critérios de exclusão:

a) Os que não retornam após 60 dias da primeira consulta do início do tratamento (abandono primário).

b) Pacientes com insuficiência renal (creatinina $>2,0 \mathrm{mg} /$ dl) (nota 1) ou hepática (TGO > 3 vezes o valor normal) no momento da seleção ou que desenvolvam o quadro clínico durante o tratamento.

c) Ó bitos até o terceiro mês de tratamento, ou após este por causa comprovadamente não relacionada a doença.

d) As gestantes, seja na seleção ou durante o tratamento.

e) Pacientes que, a qualquer momento, voluntariamente, passem a discordar do termo de consentimento e normas do protocolo.

Os pacientes que já tiverem utilizado drogas alternativas, particularmente as que serão usadas no estudo ou similares, 
só serão efetivamente arrolados em um dos grupos após teste de sensibilidade específico para as drogas usadas (nota 2).

Custo: 0 financiamento será através de recursos do PNCT e do Centro de Referência Prof. Hélio Fraga. Estima-se um custo por paciente tratado ambulatorialmente, por $12 \mathrm{me}$ ses, incluindo medicamentos, consultas médicas e de enfermagem, visitas domiciliares, exames radiológicos e laboratoriais de rotina, sem intervenções outras, da ordem de $\mathrm{R} \$$ $6.500,00$.

\section{Intercorrências:}

a) Gravidez - As pacientes originalmente arroladas após teste de gravidez negativo ( $T$ IG ou $\beta H C G$ ) que venham a apresentar-se grávidas no decurso do tratamento terão o esquema suspenso, dado os efeitos adversos e teratogênicos conhecidos das drogas em uso e eventuais efeitos e riscos desconhecidos desta associação medicamentosa. Permanecerão sob acompanhamento clínico até o parto.

b) Positividade para HIV - Os pacientes originalmente arrolados com sorologia para HIV negativa que vierem a se apresentar positivos no decurso do tratamento terão seu esquema continuado, com monitoramento clínico e laboratorial freqüente, para detecção precoce de eventuais efeitos adversos desconhecidos desta associação de drogas. Os esquemas antivirais utilizados concomitantemente serão compatíveis com os medicamentos do protocolo.

(Obs.: Os casos soropositivos tratados nesta etapa do estudo não utilizaram inibidores da protease, constantes dos "coquetéis" ora padronizados no país).

c) Outras - Q ueixas de distúrbios vestibulares e/ ou perda de audição, sugestivas de neuropatia do VIII par craniano, exigirão exame de audiometria e ajuste de doses, quando for 0 caso.

Esquemas: Serão testados esquemas compostos de 5 drogas, conforme apresentados no quadro 1 . A quinta droga dos esquemas será o ETAMBUTOL OU a RIFABUTINA, de acordo com a sensibilidade, nesta ordem.

\section{Critérios para a avaliação dos resultados:}

Favoráveis:

a) Alta por cura para os pacientes tratados pos seis meses subseqüentes a duas culturas negativa consecutivas, cump rindo pelo menos 12 meses de tratamento, ou, b) desaparecimento dos sintomas relacionados à infecção e estabilidade das imagens por um período de seis meses, para os que não apresentarem expectoração. Para estes últimos, deve ser tentada a realização de pelo menos um exame bacteriológico de secreção pulmonar obtida por métodos invasivos ou outros (escarro induzido, lavado brônquico ou LBA).

\section{Desfavoráveis:}

a) Falência bacteriológica comprovada por cultura positiva, em duas amostras, após o sexto mês de tratamento, sem melhora clínico-radiológica.

\begin{tabular}{|c|}
\hline QUADRO 1 \\
\hline ESQ U EMA A \\
(para pacientes com sensibilidade in vitro remanescente à S) \\
\hline
\end{tabular}

ESTREPTO MICINA (S) - Apresentação: frascos de $1 \mathrm{~g}$, com diluente Via de administração: IM (eventualmente EV). Doses: $1 \mathrm{~g} /$ dia ou $0,5 \mathrm{~g} /$ dia para os > 60 anos. Modo e tempo de uso: diário de segunda a sexta-feira durante 2 meses e 2 vezes por semana por 6 meses (total: oito meses de uso).

O FLO XACINA (O FL) - A presentação: comprimidos de $200 \mathrm{mg}$ ou de $400 \mathrm{mg}$. Via de administração: VO. D oses: $600 \mathrm{mg} / \mathrm{dia}$ para os $\geq 45 \mathrm{~kg}$, $400 \mathrm{mg} / \mathrm{dia}$ para os $<45 \mathrm{~kg}$. Modo e tempo de uso: diário, em duas tomadas (2 e 1 ou 1 e 1 (se de $200 \mathrm{mg}$ ) ou 1 e meio, durante 12 ou mais meses.

TERIZID O NA (TRZ) - Apresentação: comprimidos de 250mg. Via de administração: VO. Doses: $750 \mathrm{mg} / \mathrm{dia}$ para os $\geq 45 \mathrm{~kg}, 500 \mathrm{mg} / \mathrm{dia}$ para os $<45 \mathrm{~kg}$. Modo e tempo de uso: diário, em duas tomadas (2 e 1 ou 1 e 1 ), durante 12 ou mais meses ${ }^{(*)}$.

CLO FAZIMINA (CFZ) - Apresentação: cápsulas de 50 ou de $100 \mathrm{mg}$ Via de administração: VO. Doses: $100 \mathrm{mg} / \mathrm{dia}$. Modo e tempo de uso: diário, em uma única tomada, durante 12 ou mais meses ${ }^{(*)}$.

5a D roga: RIFABUTINA (RFB) - Apresentação: cápsulas de $150 \mathrm{mg}$. Via de administração: VO. Doses: $300 \mathrm{mg} /$ dia para os $\geq 45 \mathrm{~kg}, 150 \mathrm{mg} /$ dia para os < $45 \mathrm{~kg}$. Modo e tempo de uso: diário, em uma única tomada, em jejum, durante 12 ou mais meses ${ }^{(*)}$.

ETAMBUTOL (EMB) - Apresentação: comprimidos de $400 \mathrm{mg}$. Via de administração: VO. Doses: $1.200 \mathrm{mg} / \mathrm{dia}$ para os $\geq 45 \mathrm{~kg}, 800 \mathrm{mg} /$ dia para os < $45 \mathrm{~kg}$. Modo e tempo de uso: diário, em uma única tomada durante 12 ou mais meses ${ }^{(*)}$.

(*) Prolongado por mais tempo, 6 meses após negativação da cultura.

ESQ UEMA B

(indicado para pacientes resistentes à S)

CAPREO MICINA - Apresentação: frascos de $1 \mathrm{~g}$, com diluente. Via de administração: IM (exclusivamente). Doses: $1 \mathrm{~g} / \mathrm{dia}$ ou $0,5 \mathrm{~g} / \mathrm{dia}$ para os > 60 anos. Modo e tempo de uso: diário de segunda a sextafeira durante 2 meses e 2 vezes por semana por 6 meses.

O FLOXACINA - Semelhante ao anterior.

TERIZIDONA - Semelhante ao anterior.

CLO FAZIMINE - Semelhante ao anterior.

5a D roga: RIFABU TINA (RFB) ou ETAMBUTO L (EMB) - Semelhante ao anterior.

Todos os pacientes serão tratados por 12 meses, no mínimo, em qualquer circunstância.

\section{Notas:}

1) Para os pacientes com creatinina entre 1,2 e 2,0, as doses dos medicamentos serão corrigidas pelo clearance.

2) Os pacientes com uso anterior de drogas alternativas, especialmente um dos derivados quinolônicos, porém mantendo sensibilidade às mesmas, serão arrolados e tratados e avaliados separadamente, quanto aos resultados.

3) Em caso de efeito adverso, com necessidade de suspensão de um ou dois dos medicamentos, e com possibilidade de substituição por outros aos quais haja sensibilidade pelos testes laboratoriais, o tratamento será continuado, porém os resultados serão analisados separadamente.

4) A indicação cirúrgica deve ser limitada aos pacientes com lesões localizadas e unilaterais, ou bilaterais quando um dos lados não apresentar cavitações no exame de tomografia computadorizada, e ainda quando o paciente apresentar função pulmonar que permita ressecção. A cirurgia deve ser realizada até o sexto mês, mantendo o tratamento e estes resultados serão igualmente analisados em separado. 
b) Interrupção dos medicamentos por abandono após 0 2 ㅇ mês de tratamento ou faltosos com interrup ção alternada por mais de 60 dias.

c) Toxicidade grave, definida por interrupção definitiva de qualquer um dos medicamentos em uso por efeito adverso (nota 3).

d) Ó bito relacionado à doença ou indeterminado após o terceiro mês de tratamento.

e) Os que forem submetidos a cirurgia para tratamento da tuberculose durante 0 estudo (nota 4).

f) Internados por razões clínicas, por um período maior que 30 dias e que não cumpriram a proposta de tratamento estabelecida.

\section{Exames complementares:}

a) Na seleção: Bacteriologia (direto, cultura, tipificação e sensibilidade), sorologia anti-HIV, teste de gravidez para as mulheres em idade fértil, creatinina e TGO/TGP.

b) Iniciais: Imediatamente anterior ao início do tratamento e com 15 e 30 dias: $\mathrm{Rx}$ de tórax (60 dias), Bacteriologia (direto e cultura - 30 dias); H emograma completo, glicemia, creatininemia, transaminases, bilirrubinas e outros, se indicados. (0,15 e 30 dias).

c) No decurso do tratamento: Bacteriologia (direto e cultura) - mensais; Rx de tórax - trimestrais; Laboratório: hemoglobina, transaminases e creatinina ao final dos primeiros dois meses e uma vez a cada 30 dias de tratamento, até 0 final do mesmo.

d) O utros, se necessários ou indicados.

Consultas: Os pacientes deverão receber consultas quinzenais nos dois primeiros meses de tratamento e mantidas nesta regularidade durante o tratamento. Considerando-se dificuldades logísticas de transporte e distâncias, no momento do arrolamento e durante 0 tratamento do paciente devese ter em conta o nível de adesão do mesmo, avaliando a possibilidade de vê-lo(a) uma vez ao mês, após decorridos os dois primeiros meses de tratamento.

Seguimento: Os pacientes devem ser controlados por um período mínimo de 12 meses a 2 anos após o tratamento, para controle de recidivas, com avaliação clínica, radiológica e bacteriológica de 4 / 4 meses. Para tal foram criados os mecanismos de busca ativa dos mesmos através de telegramas, telefonemas e visitas domiciliares.

Testes de sensibilidade: Deverão ser feitos no início do tratamento, no 60 mês, se a cultura for positiva ainda neste momento, ou sempre que a cultura voltar a se positivar, após perío do de negativação. Os testes de sensibilidade serão realizados pelo Laboratório do Centro de Referencia Hélio Fraga, ou pelos Laboratórios dos Institutos Clemente Ferreira ou A dolfo Lutz (São Paulo), de acordo com a padronização técnica do primeiro.

\section{Resultados}

Os resultados descritivos do estudo até maio de 1998 estão mostrados na tabela 1.

Foram arrolados 197 pacientes no período de abril de 1995 a dezembro de 1997. Por diversas razões, 10 pacientes foram excluídos da análise. Em abril de 1998, permaneciam em tratamento 36 pacientes. Assim, foram analisados 149 casos com duração média de tratamento de 14 meses sem interrupção: $68,4 \%$ homens e $31,5 \%$ mulheres, com idade média de 36,9 anos, variando de 14 a 71 anos. A prevalência de HIV foi de $1,6 \%$. 0 tempo médio entre o primeiro episódio de TB e o início de tratamento atual para TBMR foi de 3,8 anos. Quanto ao padrão radiológico, 92\% dos pacientes apresentaram lesão cavitária bilateral e $8 \%$ lesão cavitária unilateral. H ouve $8 \%$ de taxa de resistência primária e $92 \%$ de taxa de resistência secundária.

Os resultados parciais mostram que 120 pacientes $(79,5 \%)$ negativaram a cultura no período de três meses. H ouve cura em $53 \%$ dos casos, falência em $31 \%$, abandono em $10 \%$ e óbito em $6 \%$.

\section{TABELA 1}

RESU LTADOS DESCRITIVOS DO ESTU DO ATÉ MAIO DE 1998

\section{DISTRIBUIÇÃO POR SEXO}

Masculino

$128-68,44 \%$

Feminino

$59-31,5 \%$

\section{DISTRIBUIÇÃO POR IDADE MÉDIA GERAL: 36,9 ANOS \\ HOMEM \\ 53,5 ANOS \\ MULHER \\ 30,3 ANOS}

\begin{tabular}{lcc}
\hline DISTRIBU IÇÃO DOS PACIENTES POR CEN TROS DE TRATAMENTO \\
BELÉM & 21 CASOS & $11,2 \%$ \\
RIO DE JANEIRO & 121 CASOS & $64,7 \%$ \\
Centro 3 & 22 CASOS & \\
Centro 2 & 41 CASOS \\
Centro 1 & 58 CASOS \\
SÃO PAULO & 45 CASOS & \\
\end{tabular}

PERFIL DE RESISTÊNCIA INICIAL

PRIMÁRIA

$15-8 \%$

SECUNDÁRIA

$172-92 \%$

PREVALÊNCIA DE HIV

$3 / 187-1,6 \%$

TEMPO MÉDIO ENTRE O PRIMEIRO EPISÓDIO DE TB E O INÍCIO DO TRATAMENTO ATUAL PARA TBMR

3,8 ANOS

\section{PADRÃO RADIOLÓGICO}

LESÃO CAVITÁRIA BILATERAL: $92 \%$

LESÃO CAVITÁRIA U NILATERAL: $8 \%$ 


\section{DISCUSSÃO}

Os resultados apresentados na presente publicação são fundamentalmente descritivos e parciais, visto que foram analisados apenas os casos de tratamento encerrado e que não sofreram tratamento estatístico que permita conclusões definitivas. Portanto, estes resultados estão sujeitos à modificação em sua análise final. Para o programa de análise e o banco de dados do estudo foi usado o software EPI INFO.

Estes resultados, entretanto, mesmo que tomando em conta diferenças metodológicas, permitem digressões comparativas com ensaios clínicos desenvolvidos com população de estudo semelhante - pacientes portadores de TBMR -, bem como as considerações que se seguem: a) a maioria dos pacientes é portadora de tuberculose multirresistente adquiri$\mathrm{da}$, resultante de mau tratamento prévio, quer por abandono precoce, quer por irregularidade na tomada de medicamentos; b) porque existe um esquema padronizado de retratamento na Brasil (E III), o critério de MR por nós utilizado foi "resistência à RH mais a uma terceira droga". 100\% do grupo aqui estudado é resistente a três ou mais drogas, configurando um perfil de resistência mais grave do que o adotado internacionalmente; c) a TBMR reproduz o perfil etário da tuberculose em geral, atingindo um grupo populacional entre as segunda e quarta décadas de vida, portanto excluindoos da atividade produtiva; d) a taxa de efetividade (aqui considerada "resultado favorável") está relacionada ao tempo de doença do paciente, isto é, quanto mais "crônico" menor a chance de cura, sobretudo quando a este fator se soma o grau de restrição funcional respiratória por destruição de tecido pulmonar, verificado quer radiológica ou tomograficamente, quer através de prova de função pulmonar; e) a taxa de falência aos esquemas testados neste grupo está relacionada a fatores de predição como: sexo masculino, uso prévio de tratamento anti-TB e lesão radiológica bilateral; f) neste grupo a multirresistência não esteve relacionada à infecção pelo HIV; g) esta taxa de resultado favorável tem sido crescente entre os pacientes arrolados mais tardiamente no estudo, estando relacionada à maior adesão ao tratamento. Poderá ser incrementada com intervenções tais como: equipe multidisciplinar para assistência integral ao paciente, consultas de enfermagem, fornecimento de vales-transporte para as consultas, visitas domiciliares aos faltosos e aos que pertençam aos subgrupos de maior risco de abandono e complementação dietética calórico-protéica; h) estas intervenções necessitam de validação na segunda etapa do estudo, iniciada em janeiro de 1998; i) o percentual de resultado favorável parece estar relacionado ao tempo de tratamento, indicando que o mínimo deveria ser de 18 e não de 12 meses; j) a cirurgia de ressecção, como complemento de tratameto, poderia ser realizada mais precocemente, nos pacientes que preenchessem os critérios para tal, conforme descrito na metodologia.

\section{REFERÊNCIAS}

1. Brasil. Ministério da Saúde. DNPS/CNCT: Manual de normas para o controle da tuberculose. 3a ed. revisada, 1988.

2. Barreto $A M W, M$ artins FM. Estudo da resistência primária no Brasil, no período de 1986 a 1988. Bol CNCT 1988;2:21-25.

3. Fiuza de Melo FA, A fiune J B, Ribeiro LHG, De Felice EAA, Castelo A. Resistência primária do Mycobacterium tuberculosis antes e depois da introdução dos esquemas 2RHZ/4RH e 3SZME/9ME em serviço de referência ambulatorial para a tuberculose em São Paulo. Informe no XXVII Congresso Brasileiro de Pneumologia e Tisiologia, Natal (RN), outubro de 1994 (in press).

4. Brasil. Ministério da Saúde. FUNASA/PNCT: Documento básico da reunião de avaliação operacional e epidemiológica do PNCT na década de 80. Brasília, 1992.

5. Gerhardt G, Miranda JA, Teixeira GM, Campos M, Natal S, Hijjar MA, Silva FEF, Dalcolmo M. Quimioterapia da tuberculose em pacientes previamente tratados com esquema $2 \mathrm{RHZ} / 4 \mathrm{RH}$. J Pneumol 1992;18(Supl 2):120.

6. Fiuza de Melo FA, Ide Neto J, Seiscento M, Pinto JA, Afiune J B. Tuberculose multirresistente. J Pneumol 1993;19:73-82.

7. Henn LA, Espina CA, Ferreira RT. Avaliação da eficácia do esquema 3-DNPS em 212 pacientes com tuberculose pulmonar. J Pneumol 1990;16(Supl 1):96-97.

8. Rizzon CFC. Informe pessoal no VIII Congresso Brasileiro de Infectologia, Porto Alegre (RS), setembro de 1994.

9. Picon PD, Rizzon CFC, Freitas TM, Azevedo SNB, Gutierrez RS. Resultados do tratamento. In: Picon PD, Rizzon CFC, Ott WP, eds. Tuberculose: epidemiologia, diagnóstico, tratamento em clínica e saúde pública. Medsi Ed. Médica e Científica, 1993.

10. Fiúza de Melo FA, Ide Neto J, Seiscento $M$, et al. Tuberculose multirresistente. J Pneumol 1993;19:73-82.

11. Fischl MA, George LD, Uttamchandani RE. Clinical presentation and outcome of patients with HIV infection and tuberculosis caused by multiple drug-resistant bacilli. Ann Intern Med 1992;117:184-190.

12. CDC-USA. National action plan to combat multidrug-resistant. Meeting the challenge of multidrug-resistent tuberculosis: summary of a conference. MMWR 1992;41(RR 11).

13. Comission de traitement de L'UICT. Considerations sur les médicaments anti-tuberculeux et recommendations sur les regimes de chiomiothérapie. Rev Fr Mal Respir 1976;4:157-162.

14. Canetti G, et al. Bulletin WHO 1969. In: Bethlem, ed. Cap. Tuberculose, 1995.

15. Fortes $A M$, Dalcolmo MP. Tuberculose multirresistente. Pulmão RJ 1996;6:68-80.

16. Pablos-Méndez $A$, Raviglione $M C$, Laszlo $A$, et al. Global surveillance for antituberculosis-drug resistence 1994-1997. N Engl J Med 1998; 338:1641-1649.

17. SBPT/MS. I Consenso Brasileiro de Tuberculose. J Pneumol 1997; 23:294-342.

18. Brasil. PNCT/Centro de Referência Prof. Hélio Fraga/M.S. - Hijjar MA, Gerhardt G, Dalcolmo MP, Fiuza de Melo FA, Kritski AL. Uma proposta de classificação e condutas para casos de tuberculose no Brasil. Recomendações de Reunião Técnica. jun/1992. J Pneumol 1993;19:1-3.

19. Fischl MA, Uttamchandani RB, Daicos GI, Poblete RB, Morena J N, Reyes RR, Boota AM, Thompson LM, Cleary TJ, Lai S. An outbreak of tuberculosis caused by multiple-drug-resistant among patients with HIV-infection. Ann Intern Med 1992;117:177-183.

20. Beck-Sague C, Dooley SW, Hutton MD, Otten J, Breeden A, Crawford J T, Pichenik AE, Woodley C, Cauthen G, J arvis WR. O utbreak of multidrug-resistant Mycobacterium tuberculosis infections in a hospi- 
tal: transmission to patients with HIV infection and staff. J AMA 1992 ; 268:1280.

21. Levy SB. Confronting multidrug resistance. A role for each of US. J AMA 1993;269:2632-2635.

22. Fiuza de Melo FM, A fiune J B. Drogas antituberculosas no Brasil: multirresistência. Ars Curandi 1992;25:51-58.

23. Riley LW. Drug-resistant tuberculosis. Clin Infect Dis 1993;17(Suppl 2):S442-446.

24. Simone MP, Dooley SW. The phenomenon of multidrug-resistant tuberculosis. In: Rossman MD, MacGregor RR, eds. Tuberculosis: Clinical management and new challenges. McGraw-Hill, Inc, 1994.

25. Penna ML, Hijjar MA, Feitosa JV, Teixeira GM, Gerhardt G, Neves J S. Resultados do tratamento de curta duração em doentes de tuberculose com recidiva. J Pneumol 1982;8(Supl):139-140.

26. Fiuza de Melo FM, Cestari F. Novas drogas para tuberculose: são necessárias? Rev Paul Med 1989;107:119-121.

27. Fiuza de Melo FM, Afiune JB. Tuberculoso crônico: um doente que precisa de ajuda... Consult Med/ APM 1992;3:36.
28. LaScolea LJ J r, Rangoonwala R. Quinolones in pulmonary tuberculosis management. Marcel Dekker Editors (special issue H oechst), 1996.

29. Simões NR, Cerqueira CM, Giudice M, Lemos ACM. Tuberculose crônica - estudo de 15 casos tratados com ciprofloxacina e amicacina. J Pneumol 1992;18(Supl 1):22.

30. Picon PD, Rizzon CFC, Freitas TM, Oliveira ESB, Tarasconi J C, Vargas $L R$. Esquemas de tuberculostáticos em pacientes com falência às "primeira e segunda linha": "terceira linha". In: Picon PD, Rizzon CFC, Ott WP, eds. Tuberculose: epidemiologia, diagnóstico, tratamento em clínica e saúde pública. Medsi Ed. Médica e Científica, 1993.

31. Afiune J B, Seiscento M, Ide Neto J, Fiuza de Melo FM, Noronha AML, Batista J, De Felice EAA, Moraes RC. Programa de assistência ao portador de tuberculose multirresistência do Instituto Clemente Ferreira - São Paulo. Informe no XXVII Congresso Brasileiro de Pneumologia e Tisiologia, Natal (RN), outubro de 1994.

32. Willcox PA, Rachman MD, Morris PG, et al. O utcome of treatment of 245 patients with multi-drug-resistant pulmonary tuberculosis. Chest 1997;112(3S) Suppl:29.

33. Goble $M$, et al. Treatment of patients with pulmonary tuberculosis resistant to isoniazid and rifampin. N Engl J Med 1993;328:527-532.

\section{MEMORIAL DA AUTORA PRINCIPAL}

Formada em 1978 pela Escola de Medicina da Santa Casa de Misericórdia, de Vitória, Espírito Santo, fez sua residência médica em Clínica Médica e Pneumologia no Hospital Raphael de Paula Souza no Rio de Janeiro, concluindo em 1981. A tua regularmente como pneumologista clínica e investigadora na área das doenças pulmonares infecciosas associadas à imunodeficiência adquiridida. Foi relatora dos Consensos Brasileiros de Pneumonia e de Tuberculose da SBPT. Doutoranda em Pneumologia, na Escola Paulista de Medicina, desenvolvendo ensaio clínico de tratamento intermitente supervisionado em pacientes com tuberculose sensível. Diretora de Assuntos Científicos da SBPT na gestão 1998-2000. 